This journal is the official publication of Bangladesh Society of Physiologists (BSP)

Web URL: www.banglajol.info/index.php/JBSP

Abstracted /indexed in Index Copernicus, Director of Open Access Journal, HINARI Index Medicus for South East Asia Region, Google Scholar, 12OR, infobse index, Open J gate, Cite factor, Scientific indexing services

pISSN-1983-1213; e-ISSN-2219-7508

\title{
Article
}

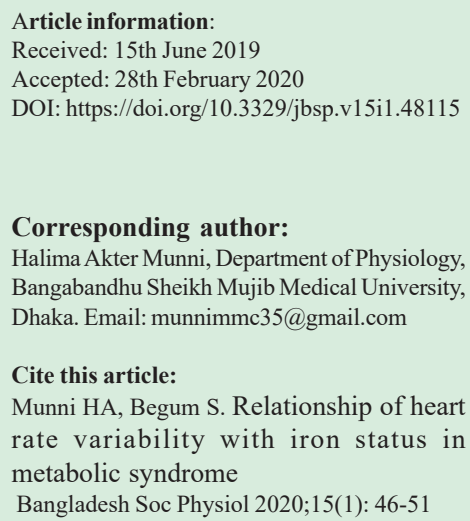

This article is open access licensed under CC BY NC SA which allows readers copy, distribute, display, and perform the work and make derivative works based on it only for noncommercial purposes.

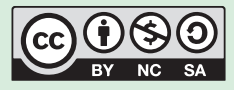

\section{Relationship of heart rate variability with iron status in metabolic syndrome}

\section{Halima Akter Munni' ${ }^{1}$, Shelina Begum ${ }^{1}$}

1. Dept. of Physiology, Bangabandhu Sheikh Mujib Medical University, Dhaka

\section{Abstract}

Background: Clustering of some most dreadful cardiovascular risk factors gives rise to metabolic syndrome (MetS). Higher iron status and impaired cardiac autonomic status may important play role in increased risk of cardiovascular morbidity in this group of patients. Objective: To observe the relationship of HRV with iron status in patients with MetS. Methods: This cross-sectional study was conducted in the Department of Physiology, Bangabandhu Sheikh Mujib Medical University (BSMMU), Shahbag, Dhaka from March, 2019 to February, 2020. For this study, 35 MetS female patients aged 25 to 45 years were enrolled in MetS group and equal number of age and sex matched apparently healthy subjects constituted control group. For evaluation of iron status, serum iron, serum ferritin, total iron binding capacity (TIBC) and transferrin saturation (Tsat) were measured by autoanalyzer. HRV was assessed by Powerlab $8 / 35$, $\mathrm{AD}$ instruments, Australia. Data were expressed as mean $\pm \mathrm{SD}$. Statistical analysis was done by Independent sample ' $t$ ' test and Pearson's correlation coefficient test as applicable. Results: In this study, resting pulse rate, systolic blood pressure (SBP), diastolic blood pressure (DBP) were significantly $(\mathrm{p}<0.001)$ higher and mean heart rate, standard deviation of the RR intervals (SDRR), mean R-R interval, standard deviation of the difference betweensuccessive $R R$ intervals (SDSD), square root of mean squared differences of successive RR intervals (RMSSD), proportion of difference of successive RR interval greater than $50 \mathrm{~ms}$ (pRR50) were significantly $(p<0.001)$ lower in MetS patients compared to control. Among the parameters of iron status, serum ferritin was significantly $(\mathrm{p} \leq<0.05)$ higher and TIBCwas significantly $(\mathrm{p}<0.05)$ lower in MetS patients in comparison to control. On correlation analysis, only the TIBC showed significant positive correlation with mean RR interval, SDRR, CVRR, SDSD, RMSSD, pRR50 ( $\mathrm{p}<0.05)$ in MetS patients. Conclusion: This study reveals that poor parasympathetic activity is related to higher iron status in metabolic syndrome patients.

Keywords: Metabolic syndrome, iron status, heart rate variability 
Introduction

Metabolic syndrome (MetS) is a cluster of major risk factors associated with cardiovascular disease (CVD) and type 2 Diabetes Mellitus. For its associated with higher risk of CVD (twofold), it is also known as cardiometabolic syndrome. According to International Diabetes Federation (IDF), MetS constitutes of central obesity plus any 2 or more of - hypertriglyceridemia, low HDL cholesterol, hypertension, fasting hyperglycemia. ${ }^{1-2}$ Cardiovascular dynamic alteration associated with MetS is manifested by autonomic dysfunction. ${ }^{3-4}$ Heart rate variability (HRV) is a valuable, widely used method to quantify autonomic function and its individual components. ${ }^{5}$ It can be useful for detecting any physiological or pathological modification in heart rate. ${ }^{6}$ HRV can be analyzed by several methods. Among them, time domain measures are simplest to perform. They can detect either the instantaneous heart rate at any specific point of time or RR interval between successive normal QRS complex of ECG. ${ }^{5}$ Time domain variables can be derived directly from RR intervals (eg. SDRR) or by statistical calculation of difference between them (eg. SDSD, RMSSD, pRR50). ${ }^{4}$ Several studies have reported lower HRV characterized by overactive sympathetic nervous system and hypoactive parasympathetic nervous system in MetS. 3,7-9

Iron, an essential trace element, plays a key role in many biological processes, including oxygen transport, DNA biosynthesis, and electron transport. Ferritin is the major iron storage protein and a small fraction of body ferritin circulates in the plasma. It is used as a clinical biomarker to assess iron status. In addition, serum iron, serum ferritin, total iron binding capacity (TIBC) and transferrin saturation (Tsat) can be measured to observe iron status. ${ }^{10-11}$ Several previous studies found considerably higher iron and ferritin in MetS. ${ }^{11-12}$ Datz and his colleagues ${ }^{13}$ observed hyperferritinemia with normal or mildly elevated transferrin saturation in this syndrome. On In contrast, some studies found no association between serum iron and MetS. ${ }^{15-16}$

Due to the ability of iron to transfer electron between ferrous and ferric forms, excess iron is detrimental- mostly via production of reactive oxygen species (ROS) (hydroxyl radical) by Fenton and Haber-Weiss reaction and therefore is a potent risk factor for cardiovascular system related morbidity and mortality. ${ }^{11-12,16}$

Despite adequate reports regarding iron overload and low HRV in MetS, little is known about the relationship between them in MetS. Exploring this relationship may be helpful in prevention, early diagnosis and effective management of CVD related morbidity in this group of patients.

\section{Methods}

This cross-sectional study was conducted in Department of Physiology, BSMMU, Shahbag, Dhaka during 2019. For this purpose, 35 female subjects with Mets diagnosed according to IDF $^{1}$ criteriaand aged 25-45 years were selected by purposive sampling from Department of Endocrinology, BSMMU and to compare with them, 35 apparently healthy subjects of similar age and sex were collected through personal contact and were enrolled in comparison group. All data were taken during proliferative phase of menstrual cycle to avoid hormonal influence. After proper review of the ethical aspects, Institutional Review Board of BSMMU approved this study procedure. Informed written consent was taken from all subjects. Subjects suffering from cardiac, respiratory, renal, thyroid disorders, any acute or chronic inflammation, menstrual abnormality were excluded from this study. Known case of hemochromatosis, thalassemia, women having history of blood transfusion within last 3 months, having history of recent major surgery or illness, taking hormonal contraceptives or iron fortified vitamin, pregnant, lactating mother, women after menopause were also excluded from this study. Detail family, menstrual, medical and dietary 
history was taken and thorough physical examination was done. Resting pulse rate, blood pressure, anthropometric parameters- waist circumference (WC), weight, height were measured. Then $9 \mathrm{ml}$ of venous blood was collected for estimation of fasting plasma glucose, lipid profile, serum SGPT, and serum creatinine in the laboratory of Department of Biochemistry and Molecular Biology and complete blood count (CBC) in Department of Laboratory Medicine by automated analyzer confirm diagnosis. Then few instructions were given for preparing the subjects for HRV. At previous night of the HRV recording day, they were requested to finish their meal by 9:00 pm and to have a sound sleep avoiding any type of stress. They were forbidden to take any sedative hypnotic medication. On the test day morning, they were advised to have a light breakfast without tea or coffee and to attend the autonomic nerve function laboratory in the Department of Physiology, BSMMU between 8:00am to 9:00 am. To adjust to the controlled laboratory environment, the subjects were advised to take rest for 15-20 minutes and during this period they were forbidden to talk, eat or drink, to perform physical or mental activity or sleep. ECG was recorded in lead II for 5 minutes by data acquisition device Power Lab 8/35 (AD Instrument, Australia). Analysis of RR interval of HRV was done by Lab chart software.

Data were expressed as Mean \pm SD. Statistical analysis was done using SPSS version 16. Independent sample ' $t$ ' test and Pearson's correlation coefficient test were done, $\mathrm{p}$ value of $£ 0.05$ was considered as statistically significant.

\section{Results}

Baseline general characteristics of all subjects are shown in Table I. Age was comparable in subjects of both groups, but according to MetS definition, WC, FPG, serum TG were significantly $(p<0.001)$ higher and serum HDL$\mathrm{C}$ was significantly $(\mathrm{p}<0.05)$ lower in group MetS in comparison to healthy subjects (Table I). MetS subjects had significantly higher resting pulse rate, SBP and DBP $(\mathrm{p}<0.001)$ compared to healthy subjects (Table II). In this study, the mean value of heart rate was significantly $(p<0.001)$ higher and mean RR interval, SDRR, CVRR, SDSD, RM SSD, pRR50 were significantly $(\mathrm{p}<0.001)$ lower in group MetS than that of healthy subjects (Table III).

Table I General characteristics in two groups $(\mathrm{N}=70)$

\begin{tabular}{lccc}
\hline Variables & MetS $(\mathrm{n}=35)$ & Control $(\mathrm{n}=35)$ & p value \\
\hline Age & $37.89 \pm 6.07$ & $35.34 \pm 5.94$ & $0.081^{\mathrm{ns}}$ \\
(years) & $(25-45)$ & $(25-45)$ & \\
WC & $95.51 \pm 5.97$ & $75.60 \pm 3.09$ & $0.000^{* * *}$ \\
$(\mathrm{~cm})$ & $(83-113)$ & $(64-79)$ & \\
FPG(mmol/L) & $8.70 \pm 3.45(5.0-16.9)$ & $5.01 \pm 0.35(4.40-5.60)$ & $0.000^{* * *}$ \\
& & & \\
HDL-C & $39.26 \pm 7.04$ & $44.34 \pm 10.14$ & $0.017^{*}$ \\
$(\mathrm{mg} / \mathrm{dL})$ & $(23-56)$ & $(31-68)$ & $0.000^{* * *}$ \\
TG & $232.89 \pm 108.47$ & $95.74 \pm 25.95$ & \\
$(\mathrm{mg} / \mathrm{dL})$ & $(98-466)$ & $(30-145)$ & \\
\hline
\end{tabular}

Data were expressed as mean $\pm \mathrm{SD}$. Values in parentheses indicate ranges; Statistical analyses were done by Independent sample ' $t$ ' test; Group A- Subjects with metabolic syndrome; Group B- Comparison group; WCWaist circumference; FPG- Fasting plasma glucose; HDL-C- High density lipoprotein cholesterol; TGTriglyceride; ns- Non significant ( $>0.05) ; * * \mathrm{*}<0.001 ;{ }^{*} \mathrm{p}<0.05$; N-Total number of subjects; $\mathrm{n}$ - Number of subjects in each group. 
Table II: Resting pulse rate and blood pressure in two groups $(\mathrm{N}=70)$

\begin{tabular}{lccc}
\hline Variables & MetS $(\mathrm{n}=35)$ & Control $(\mathrm{n}=35)$ & p value \\
\hline Pulse & $87.40 \pm 8.69$ & $73.97 \pm 7.14$ & $0.000^{* * *}$ \\
(beats $/ \mathrm{min})$ & $(65.00-104.00)$ & $(60.00-88.00)$ & \\
SBP & $132.00 \pm 13.02$ & $116.43 \pm 6.92$ & $0.000^{* * *}$ \\
$(\mathrm{~mm}$ of $\mathrm{Hg})$ & $(110.00-160.00)$ & $(100.00-125.00)$ & \\
DBP & $85.00 \pm 9.39$ & $73.14 \pm 6.07$ & $0.000^{* * *}$ \\
$(\mathrm{~mm}$ of $\mathrm{Hg})$ & $(70.00-100.00)$ & $(60.00-80.00)$ & \\
\hline
\end{tabular}

Data were expressed as mean \pm SD. Values in parentheses indicate ranges; Statistical analyses were done by Independent sample ' $t$ ' test; MetS- Metabolic syndrome; SBP- Systolic blood pressure; DBP- Diastolic blood pressure $^{* * *} \mathrm{p}<0.001 ; \mathrm{N}$ - Total number of subjects; $\mathrm{n}$ - Number of subjects in each group.

Table III: Time domain measures of HRV in two groups $(\mathrm{N}=70)$

\begin{tabular}{lccc}
\hline Variables & MetS $(\mathrm{n}=35)$ & Control $(\mathrm{n}=35)$ & p value \\
\hline Mean heart rate & $87.55 \pm 9.96$ & $75.77 \pm 7.22$ & $0.000^{* * *}$ \\
(beats/min) & $(64.90-104.20)$ & $(60.11-89.23)$ & \\
Mean RR interval & $695.66 \pm 85.86$ & $802.33 \pm 80.58$ & $0.000^{* * *}$ \\
$(\mathrm{~ms})$ & $(575.80-925.30)$ & $(673.60-1004.00)$ & \\
SDRR & $22.66 \pm 10.95$ & $46.86 \pm 16.53$ & $0.000^{* * *}$ \\
(ms) & $(6.01-49.61)$ & $(12.56-78.12)$ & \\
CVRR & $0.03 \pm 0.01$ & $0.06 \pm 0.02$ & $0.000^{* * *}$ \\
& $(0.01-0.06)$ & $(0.02-0.09)$ & \\
SDSD & $18.47 \pm 18.27$ & $43.44 \pm 21.01$ & $0.000^{* * *}$ \\
(ms) & $(2.75-72.18)$ & $(7.94-90.36)$ & \\
RMSSD & $18.49 \pm 18.34$ & $43.33 \pm 20.95$ & $0.000^{* * *}$ \\
$(\mathrm{~ms})$ & $(2.75-72.14)$ & $(7.93-90.23)$ & \\
pRR50 & $2.62 \pm 7.38$ & $21.74 \pm 19.35$ & $0.000^{* * *}$ \\
(\%) & $(0.00-35.07)$ & $(0.00-62.91)$ & \\
\hline
\end{tabular}

Data were expressed as mean $\pm \mathrm{SD}$. Values in parentheses indicate ranges; Statistical analyses were done by Independent sample ' $t$ ' test; MetS- Metabolic syndrome; SDRR- Standard deviation of all RR interval; CVRRCoefficient of variance of RR interval; SDSD- Standard deviation of successive RR interval differences between adjacent RR intervals; RMSSD- Square root of mean of squared differences of successive RR interval; pRR50Proportion of differences of successive RR interval $>50 \mathrm{~ms} ;{ }^{* * *} \mathrm{p}<0.001 ; \mathrm{n}$ - Number of subjects in each group ; N- Total number of subjects.

Volume 15 No. 1 June 2020: 46-53 
In MetS group, serum ferritin was significantly $(\mathrm{p}<0.05)$ higher and TIBC was significantly $(p<0.05)$ lower than those of control group. (Table IV).

The correlation between all time domain parameters and serum iron, serum ferritin and
Tsat were statistically non-significant $(p>0.05)$ in MetS group (Table V,VI). In correlation analysis, in MetS group, significant positive correlation was found between mean RR interval, SDRR, CVRR, pRR50 ( $\mathrm{p}<0.01)$, SDSD, RMSSD $(p<0.05)$ and TIBC (Table VI).

Table IV: Parameters of iron status in two groups $(\mathrm{N}=70)$

\begin{tabular}{lccc}
\hline Variables & MetS $(\mathrm{n}=35)$ & Control $(\mathrm{n}=35)$ & $\mathrm{p}$ value \\
\hline Iron & $61.83 \pm 28.40$ & $68.49 \pm 20.59$ & $0.266^{\mathrm{ns}}$ \\
$(\mu \mathrm{g} / \mathrm{dL})$ & $(25-144)$ & $(29-110)$ & \\
Ferritin & $122.57 \pm 143.47$ & $60.55 \pm 40.82$ & $0.017^{*}$ \\
$(\mathrm{ng} / \mathrm{mL})$ & $(7.60-526.90)$ & $(5.53-172.33)$ & \\
TIBC & $286.63 \pm 99.75$ & $325.77 \pm 49.65$ & $0.041^{*}$ \\
$(\mu \mathrm{g} / \mathrm{dL})$ & $(102-494)$ & $(215-407)$ & \\
Tsat & $24.43 \pm 15.41$ & $21.65 \pm 7.22$ & $0.337^{\mathrm{ns}}$ \\
$(\%)$ & $(5.47-69.23)$ & $(7.80-40.74)$ & \\
\hline
\end{tabular}

Data were expressed as mean \pm SD. Values in parentheses indicate ranges; Statistical analyses were done by Independent sample ' $t$ ' test;MetS- Metabolic syndrome; TIBC- Total iron binding capacity; Tsat- transferrin saturation; ${ }^{*} \mathrm{p}<0.05$; ns- N

Table V: Correlations of time domain HRV measures with serum iron and serum ferritin inMetS group $(\mathrm{n}=35)$

\begin{tabular}{lcccc}
\hline VariablesMetS & \multicolumn{2}{c}{ Serum iron } & \multicolumn{2}{c}{ Serum ferritin } \\
& r value & p value & r value & $p$ value \\
\hline Mean heart rate & 0.098 & $0.575^{\mathrm{ns}}$ & 0.103 & $0.554^{\mathrm{ns}}$ \\
Mean RR interval & 0.267 & $0.121^{\mathrm{ns}}$ & -0.140 & $0.422^{\mathrm{ns}}$ \\
SDRR & 0.119 & $0.497^{\mathrm{ns}}$ & -0.039 & $0.822^{\mathrm{ns}}$ \\
CVRR & 0.009 & $0.961^{\mathrm{ns}}$ & -0.128 & $0.465^{\mathrm{ns}}$ \\
SDSD & -0.022 & $0.902^{\mathrm{ns}}$ & 0.044 & $0.801^{\mathrm{ns}}$ \\
RMSSD & 0.030 & $0.863^{\mathrm{ns}}$ & 0.043 & $0.808^{\mathrm{ns}}$ \\
pRR50 & 0.023 & $0.895^{\mathrm{ns}}$ & -0.122 & $0.484^{\mathrm{ns}}$ \\
\hline
\end{tabular}

Statistical analyses were done by Pearson's correlation coefficient (r) test. MetS- Metabolic syndrome; SDRRStandard deviation of all RR intervals; CVRR-Coefficient of variance of RR interval; SDSD- Standard deviation of successive RR interval differences between adjacent RR intervals; RMSSD- Square root of mean of squared differences of successive RR interval; pRR50- Proportion of differences of successive RR interval >50 ms; nsNon significant ( $\mathrm{p}>0.05)$; $\mathrm{n}$ - Number of subjects. 
Table VI: Correlations of time domain HRV measures with TIBC and Tsat inMetS group ( $\mathrm{n}=35$ )

\begin{tabular}{lcccc}
\hline VariablesMetS & \multicolumn{2}{c}{ TIBC } & \multicolumn{2}{c}{ Tsat } \\
& r value & p value & r value & p value \\
\hline Mean heart rate & -0.154 & $0.377^{\text {ns }}$ & -0.038 & $0.827^{\text {ns }}$ \\
Mean RR interval & 0.501 & $0.002^{* *}$ & -0.202 & $0.243^{\text {ns }}$ \\
SDRR & 0.452 & $0.006^{* *}$ & -0.297 & $0.084^{\text {ns }}$ \\
CVRR & 0.476 & $0.004^{* *}$ & -0.289 & $0.093^{\text {ns }}$ \\
SDSD & 0.402 & $0.017^{*}$ & -0.272 & $0.114^{\mathrm{ns}}$ \\
RMSSD & 0.405 & $0.016^{*}$ & -0.246 & $0.155^{\text {ns }}$ \\
pRR50 & 0.499 & $0.002^{* *}$ & -0.198 & $0.255^{\text {ns }}$ \\
\hline
\end{tabular}

Statistical analyses were done by Pearson's correlation coefficient (r) test. MetS- Metabolic syndrome; TIBCTotal iron binding capacity; Tsat- Transferrin saturation; SDRR- Standard deviation of all RR interval; CVRRCoefficient of variance of RR interval; SDSD-Standard deviation of successive RR interval differences between adjacent RR intervals; RMSSD- Square root of mean of squared differences of successive RR interval; pRR50Proportion of differences of successive RR interval $>50 \mathrm{~ms}$; ns- Non significant ( $>0.05) ;{ }^{*} \mathrm{p}<0.05 ;{ }^{*} \mathrm{p}<0.01 ; \mathrm{n}$ number of subjects.

\section{Discussion}

In this study, significantly higher resting pulse rate, SBP and DBP in MetS patients compared to their healthy counterpart were consistent with other studies. ${ }^{3-4,7-8}$

Here, significant higher mean HR and lower mean RR interval, SDRR, SDSD, RMSSD, pRR50 in MetS patients compared to healthy subjects suggestive of lower HRV and lower parasympathetic activity in current series of MeS and this agreed with observations with other researches. ${ }^{3-4,7,17}$

The current study revealed significantly higher serum ferritin but significantly lower TIBC suggesting higher iron status/store in MetS patients.. Findings of serum ferritin were similar to other previous studies. ${ }^{11-12,18-20}$ But contrast to findings regarding TIBC in MetS. ${ }^{11,21}$ In the present study, a significant positive correlation of time domain parameters with TIBC in MetS indicates a positive relationship of cardiac parasympathetic tonic activity with iron binding capacity in MeS patients.

Obesity is assumed as the core pathogenesis of metabolic abnormalities associated with the
Mets. In obesity, iron export from enterocyte, hepatocyte, the macrophage is impaired via several mechanisms. ${ }^{22}$ Sequestration of iron leads to higher cellular and serum ferritin. As cellular store is high, this may cause decreased TIBC in MetS.

TIBC is an indirect measure of serum transferrin, which keeps the iron in a bound inactive state. In MetS, glycation may cause impaired iron binding capacity of transferrin ${ }^{23}$ and also glycation of RBC membrane protein may cause excessive lysis and release of free iron. ${ }^{24}$ As there is not enough transferrin to bind with this free iron, increased free iron concentration may cause excessive reactive oxygen species (ROS) production via Fenton and Haber- Weiss reactions. ${ }^{16,23}$ ROS may lead to reduced nitric oxide (NO) bioavailability via decreased production and increased inactivation of NO. ${ }^{25}$ Centrally acting NO modulates preganglionic parasympathetic neurons within the nucleus ambiguous and the dorsal vagal motor nucleus. NO facilitates cardio-vagal activity presynaptically via increasing release of Ach from nerve terminal. ${ }^{25-26}$ It is involved in 
cholinergic inhibition of L-type $\mathrm{Ca}^{2+}$ current $\left(\mathrm{I}_{\mathrm{Ca}, \mathrm{L}}\right)$ in cardiac pacemaker cell and thus in Ach induced bradycardia. ${ }^{26}$

\section{Conclusion}

From the results of this study, it may be concluded that cardiac parasympathetic impairment is related to iron status in metabolic syndrome.

\section{Conflict of interest-None}

\section{References}

1. International Diabetes Federation. The IDF consensus worldwide definition of the metabolic syndrome. IDF Communications 2006; 20: 1-23

2. Ma Y, Tseng PH, Ahn A, Wu MS, Ho YL, Chen MF, Peng CK. Cardiac autonomic alteration and metabolic syndrome: an ambulatory ECG-based study in a general population. Sci rep 2017; 7 (1): 1-9. DOI: $10.1038 /$ srep 44363

3. Smirnova EN, Loran EA, Shulkina SG, Podtaev SY, Trushin MV. Endothelial dysfunction and reduced heart rate variability in patients with metabolic syndrome. Ital J Med. 2018; 12(1): 51-6. DOI: $10.4081 /$ itjm.2018.847

4. Task Force of the European Society of Cardiology and the North American Society of Pacing and Electrophysiology. Heart rate variability: standards of measurement, physiological interpretation and clinical use. CIRC J 1996. 93: 1043-1065. DOI: 10.1161/ 01.CIR.93.5.1043

5. Akselrod S, Gordon D, Ubel FA, Shannon DC, Berger AC,Cohen RJ. Power spectrum analysis of heart rate fluctuation: a quantitative probe of beat-to-beat cardiovascular control. Science 1981; 213(4504): 2202. DOI: $10.1126 /$ science. 6166045

6. Aydýn G, Sarýkaya S, Turgut OO, Pahin P, Çakmak NY, Yýlmaz MB, Tandoðan Ý. Assessment of Autonomic Function in Metabolic Syndrome using Combination Heart Rate Variability and Heart Rate Turbulence. Acta Med Anatol 2013; 1(1): 21-5. DOI: 10.15824 /actamedica.5000000347

7. Stuckey MI, Kiviniemi A, Gill DP, Shoemaker JK, Petrella RJ. Associations between heart rate variability, metabolic syndrome risk factors, and insulin resistance. Appl Physiol Nutr Metab 2015. 40(7): 734-40. DOI: 10.1139/apnm-2014-0528
8. Kangas P, Tikkakoski A, Uitto M, Viik J, Bouquin H, Niemelä O, Mustonen J, Pörsti I. Metabolic syndrome is associated with decreased heart rate variability in a sex dependent manner: a comparison between 252 men and 249 women. Clin Physiol Funct Imaging 2018; 39 (2): 160-7. DOI: $10.1111 /$ cpf.12551

9. Higgins T, Eckfeldt JH, Barton JC, Doumas BT. Hemoglobin, iron and bilirubin. In: Burtis CA, Ashwood ER, Bruns DE, editors. Tietz textbook of clinical chemistry and molecular diagnostics. 2012.5 ed. USA: Elsevier Saunders. p. 985-1030.

10. Tavakoli-Hoseini N, Ghayour-Mobarhan M, Parizadeh SM, Mirhafez SR, TavallaieSh FG. Relationship between indices of iron status and metabolic syndrome in an Iranian population. J Anal Res Clin Med 2014; 2 (4): 197-205. DOI: $10.5681 /$ jarcm.2014.033

11. Leiva E, Mujica V, Sepúlveda P, Guzmán L, Núñez S, Orrego R, Palomo I, Andrews M, Arredondo MA. High levels of iron status and oxidative stress in patients with metabolic syndrome. Biol Trace Elem Res 2013; 151 (1): 1-8. DOI: 10.1007/s12011-012-9525-3

12. Datz C, Felder TK, Niederseer D, Aigner E. Iron homeostasis in the metabolic syndrome. Eur J Clin Invest 2013; 43(2): 215-24. DOI: 10.1111/eci.12032

13. Brudevold R, Hole T, Hammerstrøm J. Hyperferritinemia is associated with insulin resistance and fatty liver in patients without iron overload. PloS one 2008 ; $3(10)$ : e3547

14. Kilani N, Vollenweider P, Waeber G, Marques-Vidal P. Iron metabolism and incidence of metabolic syndrome. Nutr Metab Cardiovasc Dis 2015; 25(11): 1025-32. DOI: 10.1016/j.numecd.2015.07.005

15. Das TK, Wati MR, Fatima-Shad K. Oxidative stress gated by Fenton and Haber Weiss reactions and its association with Alzheimer's disease. Arch Neuro sci 2015; 2(2). DOI:10.5812/archneurosci.20078

16. Yoon H, Go JS, Kim KU, Lee KW. The association of serum ferritin and metabolic syndrome and metabolic syndrome score in Korean adults. Korean J Clin LabSci 2016; 48(4): 287-95. DOI: 10.15324/kjcls.2016. 48.4 .287

17. Saito I, Maruyama K, Eguchi E, Kato T, Kawamura R, Takata Y, Onuma H, Osawa H, Tanigawa T. Low Heart rate variability and sympathetic dominance modify the association between insulin Resistance and Metabolic Syndrome ${ }^{-}$The Toon Health Study. Circ J 2017: CJ-17. DOI: 10.1253/circj.CJ-17-0192

Volume 15 No. 1 June 2020: 46-53 
18. Felipe A, Guadalupe E, Druso P, Carlos M, Pablo S, Oscar C, Luis V, Diego M, Jaime R, Inés U, Federico L. Serum ferritin is associated with metabolic syndrome and red meat consumption. Oxid Med Cell Longev 2015; 2015. DOI: 10.1155/2015/769739

19. Sivasankari J, Thiruchelvan V. Serum ferritin: an early marker of insulin resistance in metabolic syndrome. Int J Sci Study 2017; 5: 59-64. DOI: 10.17354/ijss/ $2017 / 216$

20. Rutigliano I, Mancini M, Guglielmi M, D’Altilia N, Pizzolorusso I, Guida A, Di Ninno F, PettoelloMantovani M, Campanozzi A. Metabolic syndrome and iron metabolism in obese children: Is there a link?. Dig Liver Dis 2013; 45: e303. DOI: 10.1016/ j.dld.2013.08.224

21. Fernández-Real JM, McClain D, Manco M. Mechanisms linking glucose homeostasis and iron metabolism toward the onset and progression of type 2 diabetes. Diabetes care 2015; 38(11): 2169-76. DOI: $10.2337 / \mathrm{dc} 14-3082$

22. Campenhout AV, Van Campenhout CM, Lagrou AR, Manuel-y-Keenoy B. Transferrin modifications and lipid peroxidation: implications in diabetes mellitus. Free radical res 2003; 37(10): 1069-77. DOI: 10.1080/ 10715760310001600390

23. Aljwaid H, White DL, Collard KJ, Moody AJ, Pinkney $\mathrm{JH}$. Non-transferrin-bound iron is associated with biomarkers of oxidative stress, inflammation and endothelial dysfunction in type 2 diabetes. J Diabetes Complications 2015; 29(7): 943-9. DOI: 10.1016/ j.jdiacomp.2015.05.017

24. Halliwell B, Gutteridge JM. The antioxidants of human extracellular fluids. Arch Biochem Biophys 1990; 280(1): 1-8.

25. Herring N, Paterson DJ. Nitric oxide cGMP pathway facilitates acetylcholine release and bradycardia during vagal nerve stimulation in the guinea pig in vitro. J Physiol. 2001; 535(2): 507-18. DOI:10.1111/j.14697793.2001.00507.x

26. Danson EJ, Li D, Wang L, Dawson TA, Paterson DJ. Targeting cardiac sympatho-vagal imbalance using gene transfer of nitric oxide synthase. J Mol Cell Cardiol 2009; 46 (4): 482-9. DOI:10.1016/ j.yjmcc.2008.12.013 